\title{
Prevalencia de baja autoestima y nivel de resiliencia bajo, en gestantes adolescentes de poblaciones del caribe colombiano
}

\author{
Liezel Ulloque-Caamaño ${ }^{1}$, Álvaro Monterrosa-Castro ${ }^{2}$, Cindy Arteta-Acosta. ${ }^{1}$ \\ ${ }^{1}$ Médico General, candidato a Magister, Grupo de Investigación Salud de la Mujer. ${ }^{2}$ Médico Ginecólogo, Líder Grupo de \\ Investigación Salud de la Mujer, Profesor Titular. Facultad de Medicina, Universidad de Cartagena. Cartagena, Colombia.
}

\section{RESUMEN}

Introducción: El embarazo en la adolescencia es un importante problema de salud pública, con repercusiones médicas, psicológicas y sociales, relacionado con el inicio coital temprano sin protección anticonceptiva. Objetivo: Estimar la prevalencia de baja autoestima (BAE) y nivel de resiliencia bajo (NRB), en un grupo de gestantes adolescentes. Método: Estudio descriptivo transversal en gestantes adolescentes, del departamento de Bolívar, Caribe Colombiano. Se utilizó formulario para identificar características socio-demográficas de las gestantes y su compañero, historia gineco-obstétrica, estado psicoemocional con la gestación, apoyo familiar y de pareja. Se incluyó la escala de autoestima de Rosenberg y la de resiliencia de Wagnild y Young. Resultado: 406 gestantes adolescentes. Edad media: 16,5 $\pm 1,5$. La gran mayoría abandonó la escuela al quedar embarazada. La puntuación media de autoestima: $27,8 \pm 3,9$. El 76,8\% tuvieron nivel normal de autoestima y el $23,2 \%$ BAE $(p<0,05)$. La puntuación promedio de resiliencia: $125,0 \pm 17,8$. EI $8,6 \%$ tuvo nivel de resiliencia alto, $57,2 \%$ nivel de resiliencia moderado y $34,2 \% \mathrm{NRB}(p<0,05)$. Hubo correlación positiva significativa de resiliencia con: autoestima, edad de la gestante, edad del compañero, edad del primer coito, años entre primera relación sexual y edad de la gestante, años entre menarquia y primera relación coital, años entre menarquia y la edad de quedar en embarazo, años de estudio, número de controles prenatales y de ecografías acompañada por el compañero. La autoestima solo se correlacionó positiva y significativamente con la edad de la gestante. Las gestantes adolescentes que no se sentían felices o no tenían apoyo del compañero, tuvieron un significativo NRB (OR: 3,7[IC95\%:1,3-10,3]). Conclusión: La prevalencia para BAE fue de $23,2 \%$ y de $34,2 \%$ para NRB. Estos resultados señalan que se debe efectuar intervención durante el embarazo en adolescentes para elevar los niveles de autoestima y de resiliencia.

\section{PALABRAS CLAVE: Servicios de salud del adolescente, embarazo adolescente, autoestima, resiliencia}

\section{SUMMARY}

Introduction: Teenage pregnancy is a major problem of public health with medical, psychological and social consequences associated with early initiation of intercourse without sufficient contraceptive protection. Objective: To estimate the prevalence of Low Self-esteem (LSE) and Low Level of Resilience (LLR) in a group of pregnant adolescents. Methods: Cross-sectional study carried out in adolescents, in urban and rural areas of the department of Bolivar in the Colombian Caribbean pregnant. A questionnaire was applied to identify sociodemographic characteristics of the pregnant women and their partners, gynecological and obstetrical history, health history, psycho-emotional state with pregnancy, family support and partner. The Rosenberg self-esteem scale and Wagnild and Young resilience scale were included. Results: Participated 406 pregnant adolescents. The average age was: $16.5 \pm 1.5$ years. A large majority left school when they became pregnant. The average self-esteem score was $27.8 \pm 3.9$. The $76.8 \%$ of pregnant women had normal 
level of self-esteem and LSE 23.2\% $(p<0.05)$. The average score resilience was $125.0 \pm 17.8$. The 8.6\% had higher level of resilience, $57.2 \%$ moderate level of resilience and $34.2 \%$ LLR $(p<0.05)$. Statistically significant positive correlation was estimated resilience: self-esteem, age of pregnancy, age of partner, age at first intercourse, years between first intercourse and the age of pregnancy, years between menarche and first intercourse, years between menarche and age to get pregnant, years of education, number of prenatal ultrasounds and accompanied by the partner. Only self-esteem was positively correlated significantly with the age of the mother. Pregnant adolescents who did not feel happy or do not have support from partner have a significant LLR (OR 3.7[95\%Cl: 1.3-10.3]). Conclusion: The prevalence of LSE was $23.2 \%$ and $34.2 \%$ for LLR. These results indicate that intervention should be performed during adolescent pregnancy to raise levels of self-esteem and resilience.

\section{KEY WORD: Adolescent health services, adolescent pregnancy, self-esteem, resilience}

\section{INTRODUCCIÓN}

La adolescencia es una etapa vital del desarrollo humano en la cual se presentan cambios físicos y psicológicos, así como transformación en la percepción de los valores sociales. La Organización Mundial de la Salud divide adolescencia en temprana y tardía, siendo la primera entre los 10-14 y la segunda entre los 15-19 años de edad (1).

Los coitos se está iniciando a edades cada vez más tempranas, en muchas ocasiones sin el uso de medidas anticonceptivas adecuadas, trayendo como consecuencia embarazos a corta edad (1). Los embarazos en adolescentes están aumentando y se relacionan con factores biológicos, sociales y personales $(2,3)$. La Encuesta Nacional de Demografía y Salud de Colombia [ENDS-2010] (4) señaló que la prevalencia de adolescentes colombianas alguna vez embarazada, se ha incrementado desde el $13,0 \%$ en 1990 , al $17,0 \%$ en 1995 , al $19,0 \%$ en el año 2000 , al $20,5 \%$ en el 2005 y se redujo ligeramente al $19,5 \%$ en el año 2010 . También estableció que para el departamento de Bolívar, situado en el caribe colombiano, el porcentaje de adolescentes alguna vez embarazadas fue $20,0 \%$ en el 2005 y $21,3 \%$ en el 2010.

Kaye (5) señaló que los embarazos en las adolescentes, en muchos casos pueden no ser deseados y crear sentimientos negativos personales como familiares. Es generalizado el concepto que el embarazo en menores de 19 años, debe abordarse bajo la connotación de alto riesgo, involucrando aspectos médicos y sociales (2). Se ha informado mayor prevalencia de hipertensión inducida por el embarazo, restricción en el crecimiento intrauterino, parto pretérmino y recién nacido con bajo peso al nacer (6). Mayor riesgo, en comparación con adultas embarazadas, de presentar interrupción de la educación, desempleo, aislamiento social y violencia de pareja, lo cual afecta el bienestar físico, psicológico y el desarrollo social. Se ha observado que la calidad de vida de las adolescentes embara- zadas puede ser inferior a la de las adultas, en igual condición (7).

La gestación en la adolescencia puede truncar el proyecto de vida personal, especialmente si no existe apoyo familiar, social y si se es víctima de violencia intrafamiliar (5,7-10). Sin embargo, es posible afrontar dicha circunstancia y buscar la superación, es ello el concepto resiliencia psicológica. La cual también se ha definido como la capacidad que permite a una persona prevenir, minimizar o superar el daño impuesto por las adversidades de la vida y poder proyectarse hacia el futuro (11). Se ha reportado que los adolescentes tienen menor capacidad de recuperación ante las adversidades que etapas vitales mayores (8).

Al estudiarse situaciones adversas, se debiese valorar la autoestima, entendida como los sentimientos de valía personal y de respeto a sí mismo (12), ya que la baja autoestima (BAE) puede influenciar negativamente en la resiliencia. Se ha reportado que niveles altos de autoestima se asocian con mayor capacidad de resiliencia psicológica (13).

Han sido estudiadas las implicaciones médicas $(14-18)$ y las psicosociales $(2,15,19)$ del embarazo en la adolescencia. No obstante son escasos los estudios que valoran autoestima y capacidad de resiliencia, a pesar de conocerse la importancia que tienen (12,20). El objetivo fue estimar la prevalencia de BAE y el de nivel de resiliencia bajo (NRB), en un grupo de gestantes adolescentes colombianas.

\section{METODOLOGÍA}

Participantes. Estudio transversal realizado en gestantes adolescentes del departamento de Bolívar, al norte de Colombia, las cuales cumplieron los criterios de inclusión: edad entre 10 y 19 años, con cualquier edad gestacional, sin gestaciones 0 pérdidas previas y aceptación por parte del tutor y la adolescente. Quedaron excluidas aquellas que no desearon participar, las que tenían limitación sí- 
quica, física o de lectoescritura que les impidiera diligenciar el instrumento.

Médicos y enfermeras fueron capacitados como encuestadores, para identificar adolescentes embarazadas en las comunidades, visitando puerta a puerta los municipios de Arjona, Sincerin, Gambote, Rocha, Puerto Badel y Cartagena. También las captaron en el control prenatal del Hospital Local del municipio de Arjona, en la consulta de la Clínica Maternidad Rafael Calvo y en la fundación Juan Felipe Gómez Escobar, en la ciudad de Cartagena de Indias.

Instrumentos. Se aplicó cuestionario de 45 preguntas sobre características sociodemográficas de la gestante y su compañero (edad, procedencia, etnia, estado civil, núcleo familiar, número de hijos, estrato socioeconómico, nivel educativo, ocupación y hábitos), historia ginecológica y obstétrica, antecedentes de salud, estado sicológico y emocional con la gestación, apoyo familiar y de la pareja.

Escala de Autoestima de Rosenberg. Creada por M. Rosenberg en 1965. Ampliamente utilizada para la medición de la autoestima, traducida y validada en diferentes países e idiomas como francés, alemán, japonés, portugués, español. Con un Alpha Cronbach's sobre 0,75 . Es autoaplicada tipo likert y consta de diez afirmaciones sobre los sentimientos que tiene la persona acerca de ella misma, cinco son redactados de forma positiva [ítem 1,3,4,7,10] y cinco de forma negativa [ítem $2,5,6,8,9$ ], evaluando sentimientos de la persona que determinan la autoestima. Las opciones de respuestas van de 1 a 4 , siendo $1=$ muy en desacuerdo, $2=$ en desacuerdo, $3=$ de acuerdo y $4=$ muy de acuerdo. Se asigna puntaje inverso a las afirmaciones negativas. La puntuación total va de 10 (baja autoestima) a 40 (alta autoestima), puntuación más alta indica mayor autoestima. Los autores no establecieron punto de corte, sin embargo se suele considerar como rango normal, entre 25 y 35 puntos (21-23).

Escala de Resiliencia de Wagnild y Young. Creada por Wagnild y Young en 1993, con el fin de identificar cualidades personales que favorecieran la adaptación individual resiliente y conocer las características de la personalidad positiva. Puede ser aplicada de forma individual o colectiva, autoadministrable y sin tiempo límite para responder. Consta de 25 ítems escritos de forma positiva, con respuesta tipo likert que van de 1 (no estar de acuerdo en lo absoluto) a 7 (estar de acuerdo totalmente). Con la suma de todos los ítems se obtiene el valor total que oscila entre 25 y 175 puntos. Valores altos corresponden a elevada resiliencia. Un puntaje mayor a 147, indica nivel de resiliencia alta. Entre 121-146, señala nivel de resiliencia moderada. Menor a 121, nivel de resiliencia baja. Validada y traducida a varios idiomas, alfa de Cronbach, versión española: 0,93, portuguesa: 0,85, argentina: 0,72. Para la realización del trabajo se utilizó la versión argentina, la cual establece tres factores en la escala que determinan la resiliencia: capacidad de autoeficacia [ítems 1-15], capacidad de propósito y sentido de vida [ítems 16-20], y evitación cognitiva [ítems 21-25]. Los primeros dos factores se entienden como "atributos, capacidades, habilidades, o actitudes positivas que las personas necesitan poseer para afrontar eficazmente situaciones traumáticas". El tercer factor es entendido como "mecanismo de defensa necesario para reducir el impacto emocional del acontecimiento traumático y favorecer el autocontrol, la expresión emocional adecuada, evitando el desgaste emocional y canalizar adecuadamente la energía de las emociones en situaciones difíciles"(24-27).

Tamaño de la muestra. El último censo poblacional colombiano fue realizado por el Departamento Administrativo Nacional de Estadística (DANE) en el año 2005 y contó para el departamento de Bolívar, 99.191 mujeres en el rango etario 10-14 años y 90.070 en 15-19 años, para 189.261 adolescentes femeninas [www.dane.gov.co febrero-23-2013]. Para ese mismo año y para el mismo departamento, la prevalencia de adolescentes alguna vez embarazada fue del 20,0\% según la ENDS-2010 de Colombia (4), lo que permite estimar tamaño del universo de 37.852 individuos. Se calculó tamaño de muestra de 381 adolescentes gestantes embarazadas, con heterogeneidad del $50 \%$, error del $5 \%$ y nivel de confianza del $95 \%$. Para compensar formularios incorrectamente diligenciados o incompletos y considerando que algunas invitadas rehusaran participar por razones sociales, culturales o económicas se añadieron 38 (10\%) participantes. Se planeó identificar e involucrar al menos 420 adolescentes embarazadas. La muestra se seleccionó utilizando el muestreo aleatorio simple.

Análisis estadístico. Los instrumentos incompletos fueron eliminados. Los completos, tabulados en una base de datos de Microsoft Excel 2007@. El análisis estadístico se realizó con Epi-Info-7. Los datos categóricos se expresaron en frecuencias absolutas y relativas (porcentajes), los datos continuos en medias $(\overline{\mathrm{X}})$. Las medidas de dispersión fueron intervalo de confianza al $95 \%$ para los porcentajes, desviaciones estándar para medias. Las diferencias de los datos continuos fueron calculadas con Anova para variables con distribución paramétricas y con Test de Kruskal-Wallis para las no paramétricas, de acuerdo al test de Bartlett. Las diferencias porcentuales se evaluaron con Chi cuadrado $\left(x^{2}\right)$. A las dos escalas se le estableció el alpha de Cronbach's.

La medida de asociación final fue el Odds Ratio (OR) con intervalo de confianza del $95 \%$ para datos cualitativos y se estimó coeficiente de correlación de Sperman, de acuerdo la anormalidad para los datos cuantitativos. Se consideró significativo un valor $p<0,05$. 
Aspectos éticos. Estudio aprobado por el comité de ética de la Universidad de Cartagena, Colombia. Se solicitó al tutor y a la adolescente participación voluntaria y firma del consentimiento informado de acuerdo a la declaración de Helsinki. El tutor siempre fue igual o mayor a 18 años, mayoría de edad según las disposiciones legales colombianas. Estudio anónimo sin impacto sobre la integridad física y general de la participante. Se tuvieron en cuenta las normas científicas, técnicas y administrativas para la investigación en salud, establecidas en la resolución 008430 de 1993 del Ministerio de Salud de la República de Colombia.

\section{RESULTADOS}

Fueron llenados 420 formularios, 14 (3,3\%) fueron eliminados por estar incorrectamente diligenciados. Ninguna adolescente se rehusó a participar, 406 fueron estudiadas.

Edad media: 16,5 $\pm 1,5$ años. De cada diez adolescentes embarazadas: nueve en adolescencia tardía, ocho mestiza, seis residentes en áreas urbanas, nueve de estratos socioeconómicos bajos, siete en unión libre, dos vivían con su pareja y nueve habían terminado sus estudios secundarios. Tenían en promedio 9,1 1,9 años de estudios. El $76,0 \%$ estudiaba antes del embarazo y durante el embarazo el $60,0 \%$ no tenía ocupación. La mitad de las que estudiaban presentaron deserción escolar. Antes del embarazo el 80,6\% dependían económicamente de sus padres y durante la gestación el $74,6 \%$ dependían del compañero. Las características sociodemográficas se presentan en la Tabla I.

Tabla I

CARACTERÍSTICAS SOCIODEMOGRÁFICAS

$(n=406)$

\begin{tabular}{lc}
\hline Variable & $\mathrm{n}(\%)[\mathrm{IC95 \%}]$ \\
\hline Adolescencia temprana & $29(7,1)[4,9-10,2]$ \\
Adolescencia tardía & $377(92,9)[89,7-95,1]$ \\
Residencia urbana & $252(62,1)[57,1-66,7]$ \\
Residencia rural & $154(37,9)[33,2-42,8]$ \\
Estrato socioeconómico bajo - bajo & $4(1,0)[0,3-2,6]$ \\
Estrato socioeconómico bajo & $363(89,4)[85,9-92,1]$ \\
Estrato socioeconómico medio & $33(8,1)[5,7-11,3]$ \\
Estrato socioeconómico medio - alto & $5(1,2)[0,4-3,0]$ \\
Estrato socioeconómico alto & $1(0,3)[0,0-1,5]$ \\
Escolaridad Primaria (hasta 5 años) & $23(5,7)[3,7-8,5]$ \\
Secundaria (de 6 a once años) & $373(91,9)[88,6-94,2]$ \\
Técnicos (doce o trece años) & $9(2,2)[1,1-4,3]$ \\
Tecnológicos (catorce o más años) & $1(0,2)[0,0-1,5]$ \\
Convivencia con la pareja & $77(19,0)[15,3-23,1]$ \\
Convivencia con padres & $97(23,9)[19,8-28,4]$ \\
Convivencia con pareja y padres & $103(25,4)[21,2-29,9]$ \\
Convivencia con la pareja y otro familiar & $34(8,4)[5,9-11,6]$ \\
Convivencia en casa de familiar de la pareja & $80(19,7)[16,0-23,9]$ \\
Convivencia con otros familiares & $14(3,4)[1,9-5,8]$ \\
Convivencia con otras personas o amigos & $1(0,2)[0,0-1,5]$ \\
Estudiaba antes del embarazo & $311(76,6)[72,1-80,5]$ \\
Trabajaba antes del embarazo & $13(3,2)[1,7-5,5]$ \\
Estudiaba y trabajaba antes del embarazo & $13(3,2)[1,7-5,5]$ \\
No hacía nada antes del embarazo & $69(17,0)[13,5-21,0]$ \\
\hline &
\end{tabular}




\section{CONTINUACIÓN TABLA I}

\begin{tabular}{lc}
\hline Variable & $\mathrm{n}(\%)[\mathrm{IC95 \%}]$ \\
\hline Estudiaba durante el embarazo & $143(35,2)[30,6-40,1]$ \\
Trabajaba durante el embarazo & $5(1,2)[0,4-3,0]$ \\
Estudiaba y trabajaba durante el embarazo & $12(3,0)[1,6-5,2]$ \\
No hace nada durante el embarazo & $246(60,6)[55,6-65,3]$ \\
Antes del embarazo dependía de ella & $5(1,2)[0,4-3,0]$ \\
Antes embrazo dependía de la pareja & $25(6,2)[4,1-9,1]$ \\
Antes embarazo dependía de madre, padre, ambos & $327(80,6)[76,2-84,2]$ \\
Antes embarazo dependía de hermanos, abuelos & $29(7,1)[4,9-10,2]$ \\
Antes embarazo dependía de tíos & $7(1,7)[0,7-3,6]$ \\
Antes embarazo dependía de padrastro, suegros & $13(3,2)[1,7-5,5]$ \\
Durante el embarazo dependía de ella & $9(2,2)[1,1-4,3]$ \\
Durante el embarazo dependía de la pareja & $303(74,6)[70,1-78,7]$ \\
Durante el embarazo dependía de la madre, padre, ambos & $76(18,7)[15,1-22,9]$ \\
Durante el embarazo dependía de tíos & $14(3,5)[1,9-5,8]$ \\
Durante el embarazo dependía de padrastro, suegros & $4(1,0)[0,3-2,6]$ \\
\hline
\end{tabular}

Presentaron la menarquia a los $12,2 \pm 1,3$ y la primera relación coital a los $15,2 \pm 1,3$ años, para $3,1 \pm 1,6$ años desde la menarquia hasta el inicio de los coitos. Tenían 4,3 $\pm 1,7$ años desde la menarquia y $1,2 \pm 1,0$ años desde la primera relación sexual. El 56,6\% tuvieron su primer coito antes de los 15 años. La edad gestacional promedio fue $26,8 \pm 8,5$ semanas, la mitad en el tercer trimestre y ocho de cada diez nunca usaron planificación familiar antes del embarazo. Solo una $(0,2 \%)$ informó tener hábito al cigarrillo o consumir drogas psicoactivas antes y/o durante la gestación (Tabla II).

La edad media de las parejas fue de $21,0 \pm 3,3$ años, significativamente mayor que la de las adolescentes embarazadas $(16,5 \pm 1,59$ años) $(p<0,05)$. El $35,7 \%$ de los varones eran adolescentes y el $64,3 \%$ adultos, $p<0,05$. El $10 \%$ de las gestantes desconocían el nivel de escolaridad y la actividad laboral de la pareja (Tabla III).

Se observó incremento en la sensación de felicidad del $70,0 \%$ al enterarse del embarazo, al $95,8 \%$ al momento de la evaluación $(p<0,05)$. Lo mismo se observó en cuanto a la felicidad del compañero: $79,1 \%$ al $86,7 \%(p=0,0004)$. No obstante al momento de la evaluación, el $4,2 \%$ de las adolescentes no estaba feliz con el embarazo y tampoco el $13,3 \%$ de los compañeros, diferencias significativas. El apoyo familiar se incrementó desde el $79,1 \%$ hasta el $98,0 \%$. El $14,5 \%$ de las adolescentes gestantes no tenían apoyo de la pareja y el $15 \%$ informó que el compañero no estaba interesado por el embarazo.

Las puntuaciones obtenidas con escala de autoestima se presentan en la Tabla IV. El alpha de Cronbach's fue 0,588. Puntuación global promedio: $27,8 \pm 3,9$. Adolescencia temprana: $27,8 \pm 4,0$ y tardía $27,8 \pm 3,9 \quad(p=0,96)$. No hubo diferencias significativas según el trimestre del embarazo. La media de los ítems positivos: $14,0 \pm 2,5$ y para los ítems negativos: $13,8 \pm 2,1(p=0,23)$.

Tuvieron nivel de autoestima normal 312 gestantes (76,8\%) [IC95\%:72,3-80,8] y BAE 94 $(23,2 \%)[I C 95 \%: 19,2-27,6](p<0,05)$. Se sentían satisfechas con ellas mismas $(92,1 \%)$, no pensaban que no eran buenas en nada $(77,6 \%)$ y sentían que eran capaces de hacer las cosas tan bien como la mayoría de las personas $(83,0 \%)$. Cuatro de cada diez sentían o experimentaban: no tener demasiadas cosas de las que sentirse orgullosas, no tener sensación de ser una persona de valía, que no se estaban respetando a sí mismas, pensaban que eran unas fracasadas y no tenían actitud positiva hacia ellas mismas. 


\section{Tabla II
CARACTERÍSTICAS GINECOLÓGICAS Y OBSTÉTRICAS}

$n=406$

\begin{tabular}{lc}
\hline Variable & $\bar{X} \pm D E$ \\
\hline Edad de la menarquia & $12,2 \pm 1,3$ \\
Edad de primera relación sexual & $15,2 \pm 1,3$ \\
Años entre edad actual y primera relación sexual & $1,2 \pm 1,0$ \\
Años entre menarquia y primera relación sexual & $3,1 \pm 1,6$ \\
Años entre la edad actual y menarquia & $4,3 \pm 1,7$ \\
Semana de gestación & $26,8 \pm 8,5$ \\
Número de controles prenatales realizados & $3,9 \pm 2,1$ \\
Numero de ecografías realizadas & $2,1 \pm 1,4$ \\
\hline Variable & $\mathrm{n}(\%)[I C 95 \%]$ \\
\hline Con planificación antes del embarazo & $61(15,0)[11,7-18,6]$ \\
Sin planificación antes del embarazo & $345(85,0)[81,0-88,2]$ \\
Con control prenatal & $397(97,8)[95,6-98,9]$ \\
Sin control prenatal & $9(2,2)[1,0-4,3]$ \\
Trimestre de inicio de control prenatal $(n=397)$ & $138(34,8)[30,1-39,7]$ \\
Primero & $252(63,5)[58,5-68,1]$ \\
Segundo & $7(1,7)[0,7-3,7]$ \\
Tercero &
\end{tabular}

Tabla III

CARACTERISTICAS SOCIOECONOMICAS DE LA PAREJA

$n=406$

\begin{tabular}{lc}
\hline Variable & $\mathrm{n}(\%)[\mathrm{IC95 \%}]$ \\
\hline Adolescente (15 a 19 años) & $145(35,7)[31,0-40,6]$ \\
Adulto (20 a 36 años) & $261(64,3)[59,3-68,9]$ \\
Ninguna escolaridad & $3(0,7)[0,1-2,3]$ \\
Escolaridad primaria & $9(2,2)[1,0-4,3]$ \\
Escolaridad secundaria & $334(82,3)[78,1-85,7]$ \\
Escolaridad técnica & $14(3,4)[1,9-5,8]$ \\
Escolaridad tecnológico & $6(1,5)[0,6-3,3]$ \\
Escolaridad profesional & $2(0,5)[0,0-1,9]$ \\
No sabe la escolaridad & $38(9,4)[6,7-12,7]$ \\
Actual consumo de cigarrillo & $71(17,5)[13,9-21,6]$ \\
No consume cigarrillo & $324(79,8)[75,5-83,5]$ \\
No sabe si consume cigarrillo & $11(2,7)[1,4-4,9]$ \\
Nunca consumo de alcohol & $67(16,5)[13,1-20,5]$ \\
Todos los fines de semanas & $28(6,9)[4,7-9,9]$ \\
Algunos fines de semana & $297(73,2)[68,5-77,3]$ \\
No sabe si consume alcohol & $14(3,4)[1,9-5,8]$ \\
Actual uso drogas psicoactivas & $22(5,4)[3,5-8,2]$ \\
Nunca uso de drogas psicoactivas & $360(88.7)[85,0-91,5]$ \\
No sabe si consume psicoactivos & $24(5,9)[3,9-8,7]$ \\
Sin actividad laboral & $69(17,0)[13,5-21,0]$ \\
Con actividad laboral estable & $131(32,3)[27,7-37,0]$ \\
Con actividad laboral ocasional & $167(41,1)[36,3-46,1]$ \\
No sabe si tiene actividad laboral & $39(9,6)[7,0-13,0]$ \\
\hline
\end{tabular}




\section{Tabla IV \\ ESCALA DE AUTOESTIMA DE ROSENBERG}

$\mathrm{n}=406$

\begin{tabular}{|c|c|c|c|c|c|c|}
\hline & ÍTEM & $\bar{X} \pm D E$ & $\begin{array}{c}\text { Muy de } \\
\text { Acuerdo } \\
(\%)\end{array}$ & $\begin{array}{c}\text { De } \\
\text { Acuerdo } \\
(\%)\end{array}$ & $\begin{array}{c}\text { En } \\
\text { desacuerdo } \\
(\%)\end{array}$ & $\begin{array}{c}\text { Muy en } \\
\text { desacuerdo } \\
(\%)\end{array}$ \\
\hline 1. & En general estoy satisfecha conmigo misma & $3,3 \pm 0,6$ & 43,1 & 49,0 & 6,7 & 1,2 \\
\hline 2. & A veces pienso que no soy buena en nada & $2,9 \pm 0,7$ & 4,7 & 17,7 & 55,4 & 22,2 \\
\hline 3. & $\begin{array}{l}\text { Tengo la sensación de que poseo algunas } \\
\text { buenas cualidades }\end{array}$ & $2,6 \pm 0,9$ & 15,5 & 44,8 & 24,4 & 15,3 \\
\hline 4. & $\begin{array}{l}\text { Soy capaz de hacer las cosas también como la } \\
\text { mayoría de las personas }\end{array}$ & $3,0 \pm 0,6$ & 25,9 & 57,1 & 15,8 & 1,2 \\
\hline 5. & $\begin{array}{l}\text { Siento que no tengo demasiadas cosas de las } \\
\text { que sentirme orgullosa }\end{array}$ & $2,5 \pm 0,8$ & 9,6 & 31,8 & 47,8 & 10,8 \\
\hline 6. & A veces me siento realmente inútil & $3,1 \pm 0,7$ & 3,0 & 13,3 & 50,0 & 33,7 \\
\hline 7. & $\begin{array}{l}\text { Tengo la sensación de que soy una persona de } \\
\text { valía, al menos igual que la mayoría de la gente }\end{array}$ & $2,3 \pm 0,9$ & 8,9 & 42,6 & 24,4 & 24,1 \\
\hline 8. & Ojala me respetara más a mí mismo & $2,5 \pm 0,8$ & 12,3 & 32,5 & 41,4 & 13,8 \\
\hline 9. & $\begin{array}{l}\text { En definitiva, tiendo a pensar que soy una } \\
\text { fracasada }\end{array}$ & $2,5 \pm 1,0$ & 19,1 & 22,2 & 38,7 & 19,5 \\
\hline 10. & Tengo una actitud positiva hacia mí misma & $2,6 \pm 1,0$ & 26,4 & 32,8 & 18,7 & 22,2 \\
\hline
\end{tabular}

La Tabla $V$ presenta la puntuación de la Escala de Resiliencia. Alpha de Cronbach's: 0,862. Puntuación promedio: 125,0 $\pm 17,8$. Adolescencia temprana y tardía, $114,3 \pm 22,3$ vs $125,9 \pm 17,2$ respectivamente $(p=0,0006)$. No hubo diferencia significativa de la resiliencia según trimestre gestacional. Nivel de resiliencia alto en 35 gestantes adolescentes (8,6\%) [IC95\%: 6,1-11,8], moderado en $232(57,2 \%)$ [IC95\%: 52,1-61,9] y NRB en 139 (34,2\%) [IC95\%: 29,6-39,1] (p<0,05).

El factor de resiliencia denominado capacidad de autoeficacia presentó mayor puntuación $78,0 \pm 11,8$, seguido de capacidad de propósito y sentido de vida $24,9 \pm 4,6$. Finalmente el factor evitación cognitiva fue $22,0 \pm 4,3(p<0,05)$.

Hubo diferencia significativa de la media de autoestima de acuerdo a la resiliencia $(p=0,0072)$. NRB: $26,9 \pm 3,6$, nivel de resiliencia moderada: $28,2 \pm 3,8$ y nivel de resiliencia alta: $29,1 \pm 5,5$, por tanto fue mayor la puntuación de autoestima en el nivel de resiliencia alta. Por el contrario según el nivel de autoestima, no se observó diferencia significativa en la puntuación del nivel de resiliencia.
Para BAE: $121,7 \pm 20,7$ y para alta autoestima: $126,0 \pm 16,8(p=0,10)$. De los que tenían BAE: $40,3 \%$ tuvieron NRB, $52,1 \%$ moderada y $7,5 \%$ alta. De las que tuvieron autoestima normal, el $32,4 \%$ presentaron NRB; $58,6 \%$ moderada y $9,0 \%$ alta.

Se observó correlación positiva, estadísticamente significativa de resiliencia con: autoestima, edad de la gestante, edad del compañero, edad de la primera relación sexual, años entre la primera relación sexual y la edad de la gestante, años entre la menarquia y la primera relación sexual, años entre la menarquia y la edad de quedar en embarazo, años de estudio, número de controles prenatales y número de ecografías acompañada por el compañero. La autoestima solo se correlacionó positivamente de forma significativa con la edad de la gestante (Tabla VI).

Las gestantes adolescentes que no se sentían felices tuvieron 3,7 veces más NRB [IC95\%: 1,3$10,3]$, igual se observó en las que no tenían apoyo del compañero durante el embarazo [IC95\%: 2,06,5] (Tabla VII). 
Tabla V

ESCALA DE RESILIENCIA DE WAGNILD Y YOUNG $\mathrm{n}=406$

\begin{tabular}{|c|c|c|}
\hline & ÍTEM & $\bar{X} \pm D E$ \\
\hline 1. & Usualmente puede ver una situación desde varios punto de vista & $4,8 \pm 1,4$ \\
\hline 2. & La confianza en mí misma me permite pasar los tiempos difíciles & $5,3 \pm 1,4$ \\
\hline 3. & Soy decidida & $5,4 \pm 1,4$ \\
\hline 4. & Usualmente manejo los problemas de distintos modos & $4,6 \pm 1,6$ \\
\hline 5. & Siento que puedo manejar varias cosas a la vez & $4,3 \pm 1,6$ \\
\hline 6. & Cuando estoy en una situación difícil generalmente encuentro salida & $5,1 \pm 1,4$ \\
\hline 7. & Soy capaz de hacer las cosas por mí misma sin depender de los demás & $5,2 \pm 1,5$ \\
\hline 8. & Usualmente encuentro cosas de que reírme & $5,9 \pm 1,1$ \\
\hline 9. & En una emergencia soy alguien en quien las personas pueden confiar & $5,4 \pm 1,1$ \\
\hline 10. & Siento orgullo de haber logrado cosas en mi vida & $5,5 \pm 1,3$ \\
\hline 11. & He podido atravesar situaciones difíciles, porque he experimentado dificultades antes & $4,6 \pm 1,6$ \\
\hline 12. & Me entiendo a mí misma si lo creo necesario & $5,4 \pm 1,2$ \\
\hline 13. & A veces yo hago cosas quiera o no & $4,4 \pm 1,6$ \\
\hline 14. & Mantengo interés por las cosas & $5,2 \pm 1,4$ \\
\hline 15. & Mi vida tiene sentido & $6,2 \pm 1,1$ \\
\hline 16. & Generalmente tengo energía para hacer aquello que tengo que hacer & $5,3 \pm 1,3$ \\
\hline 17. & Soy autodisciplinada & $4,7 \pm 1,6$ \\
\hline 18. & Mantengo el interés en aquellas cosas importantes para mi & $5,5 \pm 1,1$ \\
\hline 19. & Cuando hago planes los llevo a cabo hasta el final & $4,9 \pm 1,5$ \\
\hline 20. & Acostumbro a tomar las cosas sin mucha preocupación & $4,2 \pm 1,6$ \\
\hline 21. & Soy amigable conmigo misma & $5,6 \pm 1,3$ \\
\hline 22. & Hago las cosas de a una por vez & $4,4 \pm 1,4$ \\
\hline 23. & Rara vez me pregunto sobre el objetivo de las cosas & $4,3 \pm 1,6$ \\
\hline 24. & No insisto en cosas en las que no puedo hacer nada al respecto & $4,5 \pm 1,6$ \\
\hline 25. & Me siento cómoda si hay gente que no me agrada & $3,1 \pm 1,9$ \\
\hline
\end{tabular}

\section{CORRELACION ENTRE AUTOESTIMA Y RESILIENCIA CON VARIABLES CUANTITATIVAS}

\begin{tabular}{|c|c|c|c|c|}
\hline \multirow[b]{2}{*}{ Variable } & \multicolumn{2}{|c|}{ Autoestima } & \multicolumn{2}{|c|}{ Resiliencia } \\
\hline & rho & Valor $\mathrm{p}$ & rho & Valor $\mathrm{p}$ \\
\hline Autoestima & - & - & $0,166^{\star}$ & 0,001 \\
\hline Resiliencia & $0,166^{\star}$ & 0,001 & - & - \\
\hline Edad de la gestante & $0,126^{\star}$ & 0,011 & $0,263^{*}$ & 0,000 \\
\hline Menarquia & $-0,009$ & 0,860 & 0,089 & 0,074 \\
\hline Edad 1ra relación sexual & 0,090 & 0,069 & $0,213^{*}$ & 0,000 \\
\hline Años desde 1ra relación sexual & 0,064 & 0,197 & $0,127^{\star}$ & 0,011 \\
\hline Años entre menarquia y 1 ra relación sexual & 0,078 & 0,116 & $0,101^{*}$ & 0,041 \\
\hline Años entre menarquia y edad actual & 0,096 & 0,052 & $0,155^{\star}$ & 0,002 \\
\hline Semana de gestación & 0,088 & 0,076 & 0,069 & 0,167 \\
\hline Años de estudio & 0,086 & 0,084 & $0,242^{*}$ & 0,000 \\
\hline Índice de masa corporal & $-0,032$ & 0,522 & 0,096 & 0,053 \\
\hline Semana del 1er control prenatal & 0,042 & 0,399 & 0,028 & 0,571 \\
\hline Numero controles prenatales & 0,032 & 0,516 & 0,043 & 0,392 \\
\hline Numero de control prenatal con el compañero & 0,054 & 0,279 & $0,140^{*}$ & 0,005 \\
\hline Numero de ecografías & $-0,021$ & 0,678 & 0,081 & 0,103 \\
\hline Numero de ecografías con el compañero & $-0,036$ & 0,469 & $0,166^{\star}$ & 0,001 \\
\hline Edad del compañero & 0,086 & 0,082 & $0,158^{*}$ & 0,001 \\
\hline
\end{tabular}

\footnotetext{
(*) Estadísticamente significativos
} 


\section{Tabla VII \\ ASOCIACIÓN ENTRE AUTOESTIMA Y RESILIENCIA CON SENTIRSE FELIZ O APOYO DEL COMPAÑERO}

\begin{tabular}{|c|c|c|c|c|c|c|c|c|}
\hline \multirow[b]{2}{*}{ Variable } & \multicolumn{4}{|c|}{ Autoestima } & \multicolumn{4}{|c|}{ Resiliencia } \\
\hline & $\begin{array}{c}\text { Baja } \\
\text { n (\%) }\end{array}$ & $\begin{array}{c}\text { Normal } \\
\mathrm{n}(\%)\end{array}$ & $\begin{array}{c}\text { Valor } \\
\mathrm{p}\end{array}$ & OR & $\begin{array}{c}\text { Baja } \\
\text { n (\%) }\end{array}$ & $\begin{array}{c}\text { Moderada } \\
\text { y Alta } \\
\text { n (\%) }\end{array}$ & $\begin{array}{c}\text { Valor } \\
p\end{array}$ & OR \\
\hline \multicolumn{9}{|c|}{$\begin{array}{l}\text { Sentirse feliz } \\
\text { ahora }\end{array}$} \\
\hline No & $6(35,3)$ & $11(64,7)$ & 0,22 & $1,8[0,6-5,1]$ & $11(64,7)$ & $6(35,3)$ & 0,006 & $3,7[1,3-10,3]$ \\
\hline $\mathrm{Si}$ & $88(22,6)$ & $301(77,4)$ & & 1 & $128(32,9)$ & $261(67,1)$ & & 1 \\
\hline \multicolumn{9}{|c|}{$\begin{array}{l}\text { Apoyo familiar } \\
\text { ahora }\end{array}$} \\
\hline No & $2(25,0)$ & $6(75,0)$ & 0,90 & $1,1[0,2-5,5]$ & $5(62,5)$ & $3(37,5)$ & 0,08 & $3,2[0,7-13,9]$ \\
\hline $\mathrm{Si}$ & $92(23,1)$ & $306(76,9)$ & & 1 & $134(33,7)$ & $264(66,3)$ & & 1 \\
\hline \multicolumn{9}{|l|}{$\begin{array}{l}\text { Apoyo del } \\
\text { compañero } \\
\text { ahora }\end{array}$} \\
\hline No & $11(18,6)$ & $48(81,4)$ & 0,37 & $0,7[0,3-1,4]$ & $36(61,0)$ & $23(39,0)$ & 0,00 & $3,7[2,0-6,5]$ \\
\hline $\mathrm{Si}$ & $83(23,9)$ & $264(76,1)$ & & 1 & $103(29,7)$ & $244(70,3)$ & & 1 \\
\hline \multicolumn{9}{|c|}{$\begin{array}{l}\text { Estrato so- } \\
\text { cioeconómico }\end{array}$} \\
\hline Bajo & $82(22,3)$ & $285(77,7)$ & 0,23 & $0,6[0,3-1,3]$ & $124(33,8)$ & $243(66,2)$ & 0,55 & $0,8[0,4-1,6]$ \\
\hline Medio/Alto & $12(30,8)$ & $27(69,2)$ & & 1 & $15(38,5)$ & $24(61,5)$ & & 1 \\
\hline
\end{tabular}

\section{DISCUSIÓN}

Se estimó prevalencia de BAE de $23,2 \%$ y de NRB de $34,2 \%$ en adolescentes embarazadas colombianas, donde la mitad de todas presentaron deserción escolar, igual a lo señalado por WilsonMitchell (28), quien observó que el $57 \%$ de las adolescentes discontinuaron los estudios por el embarazo. Esa es una de las implicaciones del embarazo en la adolescencia. El abandono escolar inicia una cascada de eventos que conllevan pérdida de oportunidades y establecimiento de circunstancias que llevan a marginación social, subdesarrollo y pobreza $(3,7,10)$.

Uno de los factores relacionados con el embarazo en adolescentes, es el inicio temprano de coitos sin método de planificación (29). Se encontró $15,2 \pm 1,3$, años, similar a lo señalado por Heavey (30), que reportó 14,5 años. Monterrosa (15) cita estudios donde encontraron que el $36 \%$ de madres adolescentes iniciaron coitos a los 16 años, 25\% a los 15 , el $10 \%$ a los 14 y el $3 \%$ entre $11-13$ años. En Colombia la ENDS-2010, informó que entre jóvenes con 20-24 años, el 14\% tuvo primer coito antes de 14 años, $60 \%$ antes de 18 años y $82 \%$ antes de 20 años (31). Arrieta (2) estudiando escolares de Colombia, también encontró que quince años fue la edad de inicio coital.
El inicio precoz coital se debe a la maduración biológica, sicológica y sexual, expresada por la menarquia y mediada por mecanismos que llevan al despertar sexual (15). Se encontró que la menarquia se presentó a los 12,2 $\pm 1,3$ años, similar a otros estudios latinoamericanos $(9,10)$, considerando que dentro del año siguiente se hace regular la ovulación, es de esperar a los trece años la posibilidad gestacional si no existe protección adecuada. Ello fue observado, que el tiempo entre el inicio coital y embarazo fue 1,2 $\pm 1,0$ años. Además, el embarazo se sucederá si no existe regulación sobre los estímulos eróticos que el adolescente recibe (15) o si carece de elementos educativos para la toma de decisiones.

La carencia de planificación quedó explicita, el $85 \%$ nunca utilizaron métodos antes del embarazo, indicador de la carencia de efectividad de los programas de salud sexual. Salazar encontró lo mismo, el $16,6 \%$ de las adolescentes usaban métodos de planificación antes del embarazo (8) y Heavey reportó que el $82 \%$ de los adolescentes no los utilizaban (30). Cifras inferiores reporta Ximenes (32), encontró que $12,9 \%$ de las adolescentes no utilizaron anticoncepción antes de la gestación. La ENDS-2010, reportó que entre adolescentes unidas el $39,5 \%$ no utilizaban anticoncepción y entre las no unidas el $20,8 \%(4,31)$. 
La edad de las parejas fue significativamente mayor a la de las embarazadas, siendo adultas el $64,3 \%$ de las parejas. Ello también se observa en otro estudio en que la edad de las gestantes adolescentes fue de $17,2 \pm 1,4$ años y sus parejas $22,0 \pm 4,0$ años (8). Observación similar reporta Wilson-Mitchell que observó que la edad promedio de las adolescentes fue 15,6 $\pm 0,9$ años y el $75 \%$ de sus parejas eran adultas (28). Gutiérrez reportó que $65 \%$ de las parejas de las adolescentes tenían más de veinte años (33).

El 70,0\% informaron sentirse felices al momento de enterarse del embarazo, sensación de felicidad que se incrementó y el 95\% manifestaron estar felices al momento de la evaluación. Cifras mayores reportó Blake, que encontró que el $41 \%$ de las embarazadas se encontraban muy felices, $40 \%$ moderadamente felices y $19 \%$ eran infelices (34). Esas cifras son superadas por Jayasvasti, que señaló que el $42 \%$ de las embarazadas tenían nivel de felicidad media y felicidad alta en el $57 \%$, y ninguna refirió ser infeliz (35). La felicidad con el embarazo, pudiese guardar relación con factores culturales, imaginarios ancestrales acerca del papel de la mujer como madre o incluso sentimientos referentes a la maternidad (36).

El apoyo familiar a la gestante fue $98,0 \%$, mayor al observado por Contreras (36), que informa que el $73,4 \%$ de puérperas adolescentes tuvo apoyo de la familia; ocho de cada diez parejas apoyaron a la gestante desde que se enteraron del embarazo, igual en las mujeres en postparto, donde el 70,7\% refirió que sus parejas les "ayudaron bastante" y $74,1 \%$ se "sintieron amadas por sus parejas" (36).

En el embarazo se puede experimentar modificaciones en la autoestima según ansiedad y depresión; de la primera se ha observado un episodio en el $37,9 \%$ de los embarazos y de la segunda en el $25,4 \%$. Las gestantes que no presentan síntomas de ansiedad ni depresión, tienen mayor autoestima con diferencia estadística significativa. De igual forma, BAE es factor para la aparición de esos síntomas psicológicos. Las gestantes con BAE no tienen preparación para enfrentar los factores estresantes del embarazo (37).

La mayoría de las estudiadas tuvieron autoestima normal, similar a lo encontrado por Overbeek (38). Debe preocupar que el $23,2 \%$ de las adolescentes embarazadas estudiadas presentaron BAE. Ulloa reportó datos similares, 27\% BAE, 63\% moderada y $9 \%$ alta (39). Cifras más altas señala Maçola (40), que reportó que el $60,6 \%$ de las gestantes mayores de veinte años presentaron autoestima insatisfactoria. Ceballos en adolescentes embarazadas encontró que el 31,8\% tenían BAE, $59,1 \%$ normal y $9,1 \%$ autoestima alta (41). En estudiantes universitarios el $15,7 \%$ tenían BAE (42). Cogollo (43) reportó en adolescentes cartageneros una BAE de $23,2 \%$.
La puntuación media de la escala de autoestima fue de $27,8 \pm 3,9$, menor que lo señalado por Rojas-Barahona, en adultas-jóvenes chilenas (21). En ambos estudios los ítems "ojala me respetara más a mí misma" y "siento que no tengo demasiadas cosas de las que sentirme orgullosa", tuvieron valores bajos, mientras que en los otros ítems, las adultas tuvieron mejor puntuación que las adolescentes gestantes. Así mismo, las últimas tuvieron peor puntuación de autoestima al compararlas con universitarias en evaluaciones de Tras (44) y Martin-Albo (45).

En el estudio se observó correlación positiva débil entre edad y autoestima ( $r h o=0,126 ; p=0,011$ ), lo que puede explicarse por la mayor madurez emocional que se sucede con el aumento de la edad, experiencia de vida, educación, lo cual redundaría en mejor capacidad para afrontar hechos de vida, así como una actitud más positiva hacia sí misma.

La resiliencia, adaptación positiva en contextos adversos $(12,46)$ contribuye a la calidad de vida, crecimiento personal, además de amortiguar y facilitar el enfrentar con éxito el estrés (47). Involucra mecanismos neurobiológicos, cognitivos y psicológicos, con menor actividad adrenérgica y mayor actividad serotoninergica, dopaminergica y estrogénica, entre otras. Estos procesos están determinados por componentes genéticos y epigenéticos, que son los que determinan la reacción ante el estrés y la adaptación requerida, componentes de la resiliencia (48).

Resiliencia alta es fundamental para sobreponerse a las adversidades y el embarazo en la adolescencia debe ser considerado una adversidad de la vida. En el estudio se observó que el 34,2\% de las embarazadas tenían NRB y una minoría nivel alto. Morales (49) tambien encontró que los adolescentes tenían en menor proporción, resiliencia alta. Los datos obtenidos distan de Rua (24), quien señaló que los adolescentes presentan capacidad de resiliencia entre niveles medio y alto.

La puntuación de resiliencia fue menor que la encontrada por Jaramillo (50) en mujeres maltratadas o la encontrada por Rodríguez en mujeres argentinas de un amplio rango de edades (25). Salazar (8) señaló que en embarazadas se presenta NRB en importante número, por debajo de la mediana calculada, hallazgos iguales a los obtenidos en este estudio. El NRB puede acompañarse de angustia psicológica, ideación suicida y ser necesario apoyo psicológico (28).

La BAE influye negativamente en la resiliencia $(12,13,20,44)$. Morales (12) reportó correlación positiva entre autoestima y resiliencia ( $\mathrm{rho}=0,51$; $p<0,01$ ) en jóvenes con edad 18,5 $\pm 0,6$ años. Igual observación encontró Leiva (20) en adolescentes en vulnerabilidad social ( $r h o=0,56 ; p<0,01$ ). Stumblingbear-Riddle (13) informó correlación positiva débil entre autoestima y resiliencia en adolescentes 
$(r=0,38 ; p<0,01)$, así como Tras (44) en estudiantes universitarios (rho $=0,41 ; p<0,01$ ). En este estudio también se encontró correlación positiva débil entre autoestima y resiliencia en embarazadas adolescentes (rho $=0,16 ; p<0,001)$.

Se observó correlación positiva de resiliencia con: edad de la gestante, años de estudio, edad del compañero, número de controles prenatales y de asistencias a ecografías acompañada por el compañero, edad del primer coito, número de años entre primer coito y edad de la gestante, años entre menarquia y la primera relación sexual, años entre menarquia y edad de embarazo. Resultados similares son señalados por otros investigadores $(8,49,51-56)$. Todas las variables señaladas pueden ser modificadas desde programas de educación sexual que generen conciencia sobre maternidad y paternidad responsable, derechos sexuales y reproductivos, así como fundamentos en sexualidad, no desde la óptica de lo prohibitivo sino desde la toma de decisiones con responsabilidad, que involucren la participación de diferentes sectores sociales.

Se recomienda introducir o mejorar programas de educación sexual en los ámbitos familiares y escolares. Es inaceptable la alta prevalencia encontrada de BAE y NRB, lo cual obliga a intervención. Existen propuestas terapéuticas válidas para adolescentes embarazadas y fortalecer autoestima y resiliencia: familismo (53), apoyo familiar (57), apoyo social $(28,57)$, consejería grupal centrada en la persona y la sicología cognitiva Ad-Din que tiene componente espiritual y religioso (58).

Es indispensable que los jóvenes tengan proyectos de vida, que realicen actividades diarias que eleven autoestima y fortalezcan la resiliencia. EI embarazo en la adolescencia debe ser prevenido. Los entes gubernamentales tienen importante reto y deben generar políticas eficientes. La sociedad y la familia no pueden ser ajenas a la problemática.

El estudio tiene como limitaciones las propias de los estudios transversales. No se tuvieron en cuenta algunas variables como familismo, espiritualidad, magnitud del apoyo social, violencia intrafamiliar entre otras, que puedan influir sobre autoestima y resiliencia. Tiene como fortaleza acercarse a medir aspectos difíciles de estimar, como resiliencia y autoestima, es al parecer el primer estudio realizado con esos fines en adolescentes embarazadas residentes en el caribe de Colombia.

Como el embarazo en la adolescencia y los factores involucrados en su génesis, guardan relación con patrones culturales, costumbres, necesidades y entornos sociales, que hacen que su impacto cambie entre comunidades, otros estudios con otros modelos muestrales son necesarios para acercarse aún más a la medición de BAE y NRB.

\section{CONCLUSIÓN}

En un grupo de gestantes adolescentes del caribe Colombiano, la prevalencia de BAE fue $23,2 \%$ y NRB 34,2\%. La autoestima se correlacionó positivamente con la resiliencia. Estos resultados señalan que se debe efectuar intervención durante el embarazo para elevar los niveles de autoestima y de resiliencia.

FINANCIACIÓN: Universidad de Cartagena, Colombia y el Departamento Administrativo de Ciencias, Tecnología e Innovación de Colombia (COLCIENCIAS). Resolución $\mathrm{N}^{\circ} 000778$ del 2013. Proyecto ganador en la Convocatoria $\mathrm{N}^{\circ}$ 617-2013, para conformar un banco de elegibles para la formación de alto nivel para la ciencia, la tecnología y la innovación (semilleros, jóvenes investigadores y doctorados).

\section{REFERENCIAS}

1. Braine T. Adolescent pregnancy: a culturally complex issue. Bulletin of the World Health Organization. 2009;87(6):410-1.

2. Arrieta $\mathrm{H}$, Ramos E, Murillo M, Mercado K, Silgado $\mathrm{O}$, Velásquez $\mathrm{K}$, et al. Prevalencia de embarazos en adolescentes escolares en la ciudad de Cartagena. Febrero a Junio de 2010. Rev Cienc Biomed. 2010;1(2):162-7.

3. Flórez C, Soto V. Estudio a profundidad. Factores protectores y de riesgo del embarazo adolescente en Colombia. [Internet] Profamilia. 2013. Disponible en: http://www.profamilia.org.co/docs/estudios/ imagenes $/ 5 \% 20-\% 20 F A C T O R E S \% 20 P R O T E C T O-$ RES\%20Y\%20DE\%20RIESGO\%20DEL\%20EMBARAZO\%20EN\%20COLOMBIA.pdf.

4. Profamilia. Encuesta nacional de demografía y salud, 2010. [Internet] Profamilia. 2013. http://www.profamilia.org.co/encuestas/Profamilia/Profamilia/index. php?option=com_content\&view $=$ article\& $i d=62 \& \mathrm{Item}$ id $=9$.

5. Kaye D. Negotiating the transition from adolescence to mother hood: Coping with prenatal and parenting stress in teenage mothers in Mulago hospital, Uganda. BMC Public Health. 2008;8:83.

6. Klima C. Centering pregnancy: a model for pregnant adolescents. $\mathrm{J}$ Midwifery Womens Health. 2003;48(3):220-5.

7. Tas Demir S, Balci E, Günay O. Comparison of life quality of pregnant adolescents with that of pregnant adults in Turkey. Upsala J Med Sci. 2010;115:275-81.

8. Salazar D, Arroyo D, Hidalgo L, Pérez F, Chedraui P. Depressive symptoms and resilience among pregnant adolescents: a case-control study. Obstet Gynecol Int. 2010;2010:1-7.

9. Leon P, Minassian M, Borgoño R, Bustamante F. Embarazo adolescente. Rev Ped Elec. 2008:5(1):42-51.

10. Gaviria S, Chaskel R. Embarazo en adolescentes de América latina y el caribe: impacto psicosocial. Precop SCP. 2013;12(3):5-16. 
11. Ali M, Dwyer D, Vanner E, Lopez A. Adolescent propensity to engage in health risky behaviors: The role of individual resilience. Int $\mathrm{J}$ Environ Res Public Health. 2010;7(5):2161-76.

12. Morales M, González A. Resiliencia-Autoestima-Bienestar psicológico y capacidad intelectual de estudiantes de cuarto medio de buen rendimiento de liceos vulnerables. Estud Pedagóg 2014;40(1):215-28.

13. Stumblingbear-Riddle G, Romans J. Resilience among urban American Indian adolescents: exploration into the role of culture, self-esteem, subjective well-being, and social support. Am Indian Alsk Native Ment Health Res. 2012;19(2):1-19.

14. Monterrosa A, Bello A. Atención obstétrica en adolescentes menores de 15 años. Rev Colomb Obstet Ginecol. 1996;47(1):15-21.

15. Monterrosa A. Causas e implicaciones médico-sociales del embarazo en la adolescencia. Rev Colomb Obstet Ginecol. 1998;49(4):525-30.

16. Congote-Arango L, Vélez-García M, Restrepo-Orrego L, Cubides-Munévar Á, Cifuentes-Borrero R. Adolescencia como factor de riesgo para complicaciones maternas y perinatales en Cali, Colombia, 2002-2007: Estudio de corte transversal. Rev Colomb Obstet Ginecol. 2012;63(2):119-26.

17. Amaya J, Borrero C, Ucrós S. Estudio analítico del resultado del embarazo en adolescentes y mujeres de 20 a 29 años en Bogotá. Rev Colomb Obstet Ginecol. 2005;56(3):216-24.

18. Monterrosa A, Arrias M. Partos vaginales y cesáreas en adolescentes: comportamiento entre 1993 y 2005 Hospital de Maternidad "Rafael Calvo", Cartagena (Colombia). Rev Colomb Obstet Ginecol. 2007;58(2):107-14.

19. Olivera-Díaz Á, Mulett-Torres L, Luna-Díaz L. Caracterización cualitativa del embarazo en adolescentes atendidas en la clínica de maternidad Rafael Calvo en Cartagena. Colombia. Rev Cienc Biomed. 2013;4(2):262-9.

20. Leiva L, Pineda M, Encina Y. Autoestima y apoyo social como predictores de la resiliencia en un grupo de adolescentes en vulnerabilidad social. Rev Psicol. 2013;22(2):111-23.

21. Rojas-Barahona C, Zegers B, Förster C. La escala de autoestima de Rosenberg: Validación para Chile en una muestra de jóvenes adultos, adultos y adultos mayores. Rev Med Chile. 2009;137(6):791-800.

22. Espectro Autista Info. Escala de Autoestima de Rosenberg (RSES). Disponible en: http://espectroautista.info/tests/emotividad/bienestar-emocional/RSES.

23. Veselska Z, Madarasova A, Orosova O, Gajdosova B, Van Dijk J, Reijneveld S. Self-esteem and resilience: The connection with risky behavior among adolescents. Addict Behav. 2009;34:287-91.

24. Rua M, Andreu J. Validación psicométrica de las escalas de resiliencia (RS) en una muestra de adolescente portugués. Psicopatología Clínica, Legal y Forense. 2011;11:51-65.

25. Rodríguez M, Pereyra M, Gil E, Jofré M, De Bortoli M, Labiano L. Propiedades psicométricas de la escala de resiliencia versión argentina. Evaluar. 2009;9:72-82.

26. Vinaccia S, Quiceno J. Resiliencia y calidad de vida relacionada con la salud en pacientes con insuficiencia renal crónica - IRC. Revista Argentina de Clínica Psicológica. 2011; XX(3):201-11.
27. Majul E, Casari L, Lambiase S. Resiliencia: Una experiencia con adolescentes de distinto contexto socioeconómico. Rev Elect Psicol. 2012;I(1):19-40.

28. Wilson-Mitchell K, Bennett J, Stennett R. Psychological health and life experiences of pregnant adolescent mothers in Jamaica. Int $\mathrm{J}$ Environment Res Public Health. 2014;11(5):4729-44.

29. Universidad del Norte. Caracterización de los conocimientos, imaginarios culturales, creencias y normas sociales percibidas en relación con sexualidad, salud sexual y reproductiva, derechos sexuales y reproductivos, género, consumo de sustancias psicoactivas y el uso de medios y procesos de comunicación, en el marco del trabajo con redes sociales. Estudio de Línea de Base Cuantitativa y Cualitativa. Barranquilla: 2009.

30. Heavey E, Moysich K, Hyland A, Druschel C, Sill M. Female adolescents' perceptions of male partners' pregnancy desire. J Midwifery Women's Health. 2008;53(4):338-44.

31. Profamilia. Encuesta nacional de demografia y salud. Profamilia. Informe de prensa. 2010. Disponible en: http://www.profamilia.org.co/encuestas/Profamilia/ Profamilia/images/stories/documentos/ProfamiliaInforme-Prensa-2010.pdf.

32. Ximenes F, De Araujo M, Rocha J, Olm I. Gravidez na adolescência: motivos e percepções de adolescentes. Rev Bras Enferm. 2007;60(3):279-85.

33. Gutierrez T, Pascacio E, De la Cruz A, Carrasco E. Situación socio familiar y nivel de autoestima de la madre adolescente. Rev Enferm. 2002;10(1):21-5.

34. Blake S, Kiely M, Gard C, El-Mohandes A, El-Khorazaty $M$, Initiative N-D. Pregnancy intentions and happiness among pregnant black women at high risk for adverse infant health outcomes. Perspect Sex Reprod Health. 2007;39(4):194-205.

35. Jayasvasti K, Kanchanatawan B. Happines and related factors in pregnant women. J Med Assoc Thai. 2005;88(4):220-5.

36. Contreras-Pulache $\mathrm{H}$, Mori-Quispe E, Lam-Figueroa $\mathrm{N}$, Quino-Villanueva K, Espinoza-Lecca E, Yancachajlla-Apaza M, et al. Felicidad en mujeres puérperas: estudio multicéntrico en Lima Metropolitana y Callao. Rev Peru Epidemiol. 2012;16(1):1-7.

37. Makara-Studzińska M, Morylowska-Topolska J, Sygit K, Sygit M, Goździewska M. Socio-demographical and psychosocial determinants of anxiety symptoms in a population of pregnant women in the regions of central and eastern Poland. Ann Agric Environ Med. 2013;20(1):195-202.

38. Overbeek G, Zeevalkink H, Vermulst A, Scholte R. Peer victimization, self-esteem, and ego resilience types in adolescents: a prospective analysis of personcontext interactions. Soc Dev. 2010;19(2):270-84.

39. Ulloa N. Niveles de Autoestima en Adolescentes Institucionalizados. Hogar de Menores: Fundación Niño y Patria. Valdivia, II semestre 2002. [disertación] Chile: Universidad Austral de Chile; 2003.

40. Maçola L, Vale L, Carmona E. Avaliação da autoestima de gestantes com uso da Escala de Autoestima de Rosenberg. Rev Esc Enferm. 2010;4(3):570-7.

41. Ceballos G, Camargo K, Jiménez I, Requena K. Nivel de autoestima en adolescentes embarazadas en la comuna 5 de Santa Marta (Colombia). Rev Psicol. 2011;3(1):29-38. 
42. Ceballos-Ospino G, Arias-Montoya M, Romero-Barrios A, Herazo E, Oviedo-Acevedo $\mathrm{H}$, Campo-Arias A. Asociación entre orientación sexual y autoestima en estudiantes universitarios. Rev. Cienc. Biomed. 2013;4(2):270-4.

43. Cogollo-Milanes Z, Gomez-Bustamante E. Variables asociadas al inicio del consumo de cigarrillo en adolescentes estudiantes de básica secundaria de los colegios oficiales de la ciudad de Cartagena, Colombia. Aquichan. 2014;14(2):226-36.

44. Tras Z, Arslan C, Hamarta E. An examination of resilience in university students in terms of self-esteem and social self-efficacy. Int J Acad Res. 2013;5(3):325-30.

45. Martín-Albo J, Núñez J, Navarro J, Grijalvo F. The Rosenberg self-esteem scale: translation and validation in university students. Spanish J Psychol. 2007; 10(2):458-67.

46. Davydov D, Stewart R, Ritchie K, Chaudieu I. Resilience and mental health. Clin Psychol Rev. 2010;30(5):479-95.

47. Veloso C, Cuadra A, Antezana I, Avendaño R, Fuentes L. Relación entre inteligencia emocional, satisfacción vital, felicidad subjetiva y resiliencia en funcionarios de educación especial. Estudios Pedagógicos. 2013; XXXIX(2):355-66.

48. D’Alessio L. Mecanismos Neurobiológicos de la Resiliencia: Polemos; 2008. Available from: http://www. gador.com.ar/iyd/psiquiatria/pdf/resiliencia.pdf.

49. Morales M, Díaz D. Estudio comparativo de la resiliencia en adolescentes: el papel del género, la escolaridad y procedencia. Uaricha, Revista de Psicología. 2011;8(17):62-77.

50. Jaramillo-Vélez D, Ospina-Muñoz D, CabarcasIglesias G, Humphreys J. Resiliencia, espiritualidad, aflicción y tácticas de resolución de conflictos en mujeres maltratadas. Rev Salud Pública. 2005;7(3):281-92.

51. Alvarez-Aguirre A, Alonso-Castillo M, Guidorizzi A. Factores predictivos del uso de alcohol y tabaco en los adolescentes. Rev Latino-Am. Enfermagem. 2014;22(6):1056-62.

52. Álvarez L, Cáceres L. Resiliencia, rendimiento académico y variables sociodemográficas en estudiantes universitarios de Bucaramanga (Colombia). Psicol Iberoamericana. 2010;18(2):37-46.

53. Feldman J, Pittman S. Adolescent pregnancy along the Texas-Mexico border: a systematic analysis of risk and resiliency in a Mexican American population. Soc Perspec. 2008;10(1):29-52.

54. Rodriguez N, Bingham C, Paez N, Myers H. Exploring the complexities of familism and acculturation: central constructs for people of mexican origin. Am J Community Psychol. 2007;39:61-77.

55. Gómez-Azcarate E, Vera A, Ávila M, Musitu G, Vega $\mathrm{E}$, Dorantes $\mathrm{G}$. Resiliencia y felicidad de adolescentes frente a la marginación urbana en México. Psicodebate. $2014 ; 14(1): 45-68$

56. Salgado Lévano A. Felicidad, resiliencia y optimismo en estudiantes de colegios nacionales de la ciudad de lima. Liberabit. 2009;15(2):133-41.

57. Parra A, Oliva A, Sanchez-Queija I. Evolución y determinantes de la autoestima durante los años adolescentes. Anuario de Psicologia. 2004;35(3):331-46.

58. Sa'ad a F, Yusooff F, Nen S, Subhi N. The effectiveness of person-centered therapy and cognitive psychology ad-din group counseling on self-concept, depression and resilience of pregnant out-of-wedlock teenagers. Procedia - Social and Behavioral Sciences. 2014;114:927-32. 